\title{
RENAL INJURY AND NEUTROPHIL-TO-LYMPHOCYTE RATIO IN HENOCH-SCHONLEIN PURPURA
}

\author{
H. Giniès, F. Porcheret, P. Eckart \\ Centre Hospitalier Universitaire de Caen, France
}

\section{Introduction}

Henoch-Schonlein purpura (HSP) is a systemic vasculitis whose long-term prognosis is related to renal impairment.

Few biological factors are known to predict renal impairment.

The objective of this study was to evaluate the neutrophil-tolymphocyte ratio (NLR) as a predictor of kidney damage in children with HSP.

\section{Method}

114 patients with a diagnosis of HSP were included over a retrospective period of five years.

The clinical and biological data collected were those found at the time of HSP diagnosis. Renal impairment ranged from isolated proteinuria to nephrotic syndrome.

\section{Results}

Renal involvement occurred in 33 patients (29\%). In univariate analysis, the median NLR was significantly higher in cases of renal impairment (1.8 vs. 4.1, $p<0.001)$. In multivariate analysis, the risk factors for renal impairment were: age greater than eight years $(\mathrm{OR}=3, \mathrm{p}=0.02, \mathrm{Cl} 1.2$ to 7.8$)$, presence of abdominal pain (OR $=4.9, p=0.005, \mathrm{Cl} 1.6$ to 14.9$)$ and an NLR greater than $2.5(\mathrm{OR}=3.5, \mathrm{p}=0.01,1.3-1.1 \mathrm{IC})$. The combination of these three factors was found in $71 \%$ of children with renal impairment.

\section{Conclusion}

Patients with elevated NLR at the time of HSP diagnosis appear to have an increased risk of developing kidney injury and require regular monitoring.

The NLR could also be evaluated in case of relapse.

As the renal biopsy is not always enough to predict the evolution of injury, NLR included in a biological and clinical score could be studying.

\begin{tabular}{|lccc|}
\hline Clinical data & $\begin{array}{c}\text { No renal impairment } \\
(\mathrm{n}=81)\end{array}$ & $\begin{array}{c}\text { Renal impairment } \\
(\mathrm{n}=33)\end{array}$ & $\mathrm{p}$ \\
\hline Mean age & $6,4( \pm 2,9)$ & $8,6( \pm 2,9)$ & 0,01 \\
\hline Male & $42(51,9 \%)$ & $22(66,7 \%)$ & 0,148 \\
\hline $\begin{array}{l}\text { Arthritis or } \\
\text { arthralgia }\end{array}$ & $73(90,1 \%)$ & $31(93,9 \%)$ & 0,514 \\
\hline Abdominal pain & $38(46,9 \%)$ & $28(84,8 \%)$ & $<0,001$ \\
\hline
\end{tabular}

\begin{tabular}{|c|c|c|c|}
\hline \multicolumn{4}{|c|}{ Univariate comparison of CBC at diagnosis of HSP } \\
\hline & No renal involment $(n=81)$ & Renal involment $(n=33)$ & $\mathrm{p}$ \\
\hline WBC (G/L) & $10,9(8,7-13,4)$ & $12(9,2-14)$ & 0,415 \\
\hline Neutrophils (G/L) & $6,2(4,3-8,9)$ & $8,3(5,6-10,6)$ & 0,01 \\
\hline Lymphocytes (G/L) & $3,2(2,4-4,3)$ & $2,3(1,8-3,1)$ & 0,001 \\
\hline NLR & $1,8(1,2-3)$ & $4,1(2,3-5,2)$ & $<0,001$ \\
\hline
\end{tabular}

\begin{tabular}{|lcccc|}
\hline $\begin{array}{l}\text { Multivariate analysis at diagnosis of HSP } \\
\text { Risks Factors }\end{array}$ & $\begin{array}{c}\text { No renal impairment } \\
(\mathrm{n}=81)\end{array}$ & $\begin{array}{c}\text { Renal impairment } \\
(\mathrm{n}=33)\end{array}$ & $\mathrm{p}$ & $\begin{array}{l}\text { OR } \\
(\text { IC 95\%) }\end{array}$ \\
\hline Male & $42(51,9 \%)$ & $22(66,7 \%)$ & 0,894 & \\
\hline Age $>\mathbf{8 y}$ & $18(22,2 \%)$ & $18(15,8 \%)$ & 0,022 & $3(1,2-7,8)$ \\
\hline $\begin{array}{l}\text { Abdominal } \\
\text { pain }\end{array}$ & $38(46,9 \%)$ & $28(84,8 \%)$ & 0,005 & $\begin{array}{l}4,9 \\
(1,6-14,9)\end{array}$ \\
\hline NLR >2,5 & $29(35,8 \%)$ & $24(72,7 \%)$ & 0,011 & $3,5(1,3-9,1)$ \\
\hline
\end{tabular}

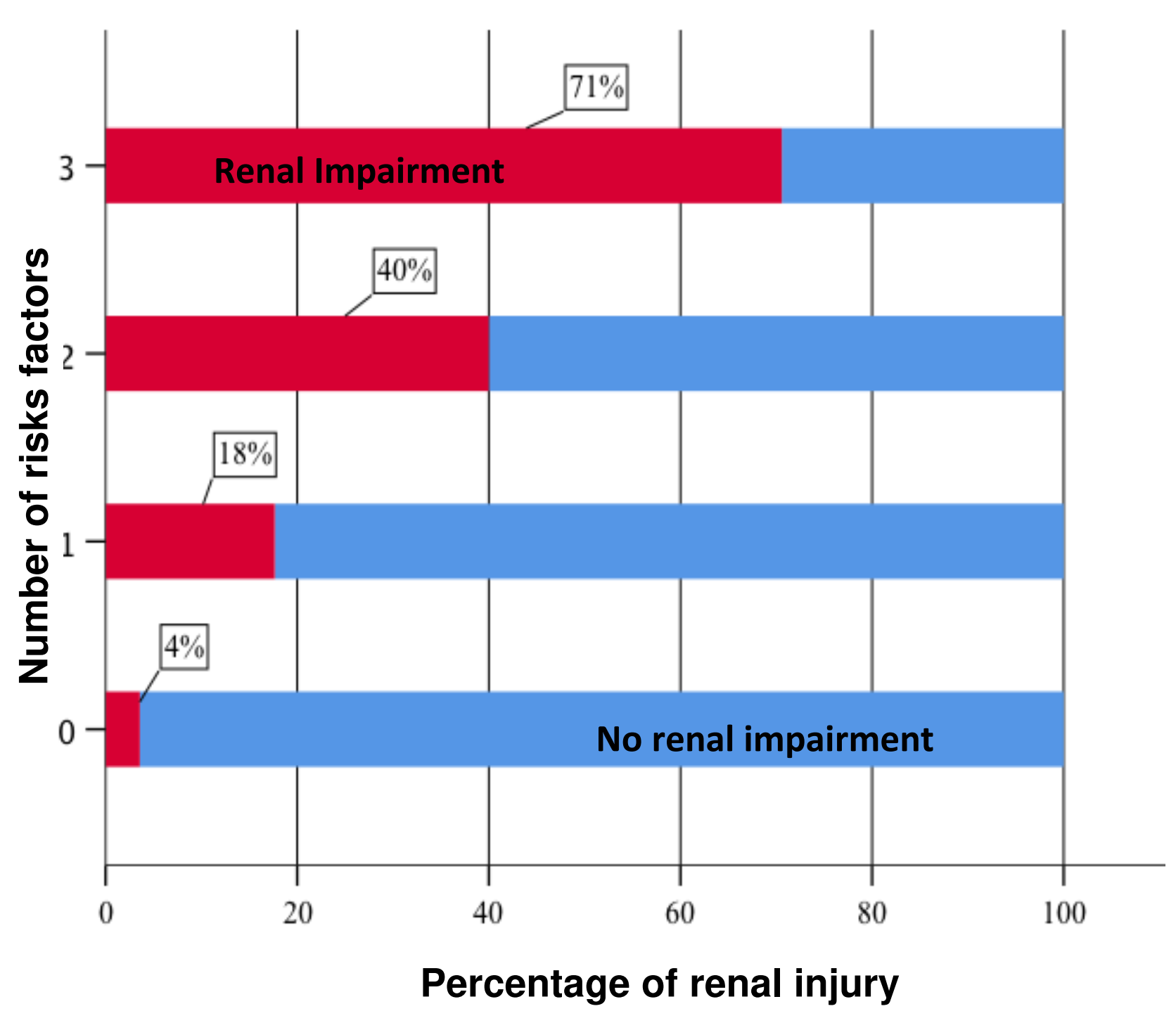

